\title{
GRB 010921: STRONG LIMITS ON AN UNDERLYING SUPERNOVA FROM THE HUBBLE SPACE TELESCOPE
}

\author{
P. A. Price, ${ }^{1,2}$ S. R. Kulkarni ${ }^{2}$ B. P. Schmidt,${ }^{1}$ T. J. Galama,${ }^{2}$ J. S. Bloom ${ }^{2}$ E. Berger,${ }^{2}$ \\ D. A. Frail, ${ }^{2,3}$ S. G. Duorgovski, ${ }^{2}$ D. W. Fox, ${ }^{2}$ A. A. Henden, ${ }^{4}$ S. Klose, ${ }^{5}$ F. A. Harrison, ${ }^{2}$ \\ D. E. Reichart, ${ }^{2}$ R. Sari, ${ }^{6}$ S. A. Yost, ${ }^{2}$ T. S. Axelrod, ${ }^{1}$ P. McCarthy, ${ }^{7}$ J. Holtzman, ${ }^{8}$ \\ J. P. Halpern, ${ }^{9}$ R. A. Kimble, ${ }^{10}$ J. C. Wheeler, ${ }^{11}$ R. A. Chevalier,${ }^{12}$ K. Hurley, ${ }^{13}$ \\ G. R. Ricker, ${ }^{14}$ E. Costa,${ }^{15}$ F. Frontera, ${ }^{16,17}$ AND L. Piro ${ }^{15}$ \\ Received 2002 July 9; accepted 2002 October 23
}

\begin{abstract}
GRB 010921 was the first HETE-2 gamma-ray burst (GRB) to be localized via its afterglow emission. The low redshift of the host galaxy, $z=0.451$, prompted us to undertake intensive multicolor observations with the Hubble Space Telescope with the goal of searching for an underlying supernova (SN) component. We do not detect any coincident SN to a limit 1.33 mag fainter than SN $1998 \mathrm{bw}$ at $99.7 \%$ confidence, making this one of the most sensitive searches for an underlying SN. Analysis of the afterglow data allows us to infer that the GRB was situated behind a net extinction (Milky Way and the host galaxy) of $A_{V} \sim 1.8$ mag in the observer frame. Thus, had it not been for such heavy extinction, our data would have allowed us to probe for an underlying $\mathrm{SN}$ with brightness approaching those of more typical Type Ib/c SNe.
\end{abstract}

Subject heading: gamma rays: bursts

\section{INTRODUCTION}

Since the discovery of gamma-ray burst (GRB) afterglows, there has been growing evidence linking GRBs to massive stars: the host galaxies of GRBs are star-forming galaxies, and the positions of GRBs appear to trace the blue light of young stars (Bloom, Kulkarni, \& Djorgovski 2002a); some of the host galaxies appear to be dusty with star formation rates comparable to ultraluminous infrared galaxies (Berger, Kulkarni, \& Frail 2001a; Frail et al. 2002).

${ }^{1}$ Research School of Astronomy and Astrophysics, Mount Stromlo Observatory, via Cotter Road, Weston, ACT 2611, Australia.

2 Palomar Observatory, MS 105-24, California Institute of Technology, Pasadena, CA 91125.

${ }^{3}$ National Radio Astronomy Observatory, P.O. Box O, Socorro, NM 87801.

${ }^{4}$ Universities Space Research Association/US Naval Observatory, Flagstaff Station, P.O. Box 1149, Flagstaff, AZ 86002-1149.

5 Thuringer Landessternwarte Tautenburg, Karl-SchwarzschildObservatorium, Sternwarte 5, 07778 Tautenburg, Germany.

6 Theoretical Astrophysics, MS 130-33, California Institute of Technology, Pasadena, CA 91125.

${ }_{7}^{7}$ Carnegie Observatories, 813 Santa Barbara Street, Pasadena, CA 91101.

8 Department of Astronomy, MSC 4500, New Mexico State University,

P.O. Box 30001, Las Cruces, NM 88003

${ }^{9}$ Columbia Astrophysics Laboratory, Columbia University, 550 West 120th Street, New York, NY 10027.

10 NASA Goddard Space Flight Center, Laboratory for Astronomy and Solar Physics, Code 681, Greenbelt, MD 20771.

${ }^{11}$ Department of Astronomy, University of Texas at Austin, RLM 15.308, Austin, TX 78712.

12 Department of Astronomy, University of Virginia, P.O. Box 3818, Charlottesville, VA 22903-0818.

13 Department of Astronomy, University of California, Space Sciences Laboratory, 601 Campbell Hall 3411, Berkeley, CA 94720.

${ }^{14}$ Center for Space Research, Massachusetts Institute of Technology, 70 Vassar Street, Building 37, Cambridge, MA 02139.

15 Istituto Astrofisica Spaziale and Fisica Cosmica, CNR, Via Gobetti, 101, 40129 Bologna, Italy.

${ }^{16}$ Istituto Astrofisica Spaziale, CNR, Area di Tor Vergata, Via Fosso del Cavaliere 100, 00133 Rome, Italy.

${ }^{17}$ Dipartimento di Fisica, Università di Ferrara, Via Paradiso 12, 44100 Ferrara, Italy.
On smaller spatial scales, there is growing evidence tying GRBs to regions of high ambient density (Galama \& Wijers 2001; Harrison et al. 2001), and at least some of the so-called dark GRBs arise in or behind regions of high extinction (Djorgovski et al. 2001a; Piro et al. 2002).

However, the most direct evidence linking GRBs to massive stars comes from observations of underlying supernovae $(\mathrm{SNe})$ and $\mathrm{X}$-ray lines. The presence of X-ray lines would require a significant amount of matter on stellar scales (e.g., Piro et al. 2000), as may be expected in models involving the death of massive stars. However, to date, these detections (e.g., Piro et al. 2000; Reeves et al. 2002) have not been made with high significance.

If GRBs do arise from the death of massive stars, then it is reasonable to expect associated SNe. The GRB-SN link was observationally motivated by two discoveries: the association of GRB 980425 with the peculiar Type Ic SN 1998bw (Galama et al. 1998; Kulkarni et al. 1998) and an excess of red light superposed on the rapidly decaying afterglow of GRB 980326 (Bloom et al. 1999). However, these two discoveries were not conclusive. SN 1998bw was never unambiguously connected with GRB 980425, and, taken at face value, the association would require GRB 980425 to be extraordinarily underenergetic as compared to all other cosmologically located GRBs. The case for GRB 980326 is weakened by the lack of a redshift for the GRB or the host galaxy. Nonetheless, the two discoveries motivated searches for similar underlying SN components. As summarized in $\S 5$, suggestions of similar red "bumps" in the light curves of various other GRB afterglows have been made (to varying degrees of confidence).

However, there is little dispute that the well-studied red bump in the afterglow of GRB 011121 is most easily explained by an underlying SN (SN 2001ke; Stanek et al. 2002; Bloom et al. 2002b; Garnavich et al. 2002). Furthermore, from radio and IR observations of the afterglow (Price et al. 2002b), there is excellent evidence that the circumburst medium was inhomogeneous with ambient density $\rho \propto r^{-2}$, as expected from a massive star progenitor 
(Chevalier \& Li 2000); here $r$ is the distance from the progenitor.

These developments are in accordance with the expectation of the "collapsar" model (Woosley 1993; MacFadyen $\&$ Woosley 1999). In this model, the core of a rotating massive star collapses to a black hole, which then accretes matter and drives a relativistic jet. Internal shocks within this jet first cause bursts of gamma rays and then subsequently result in afterglow emission as the jet shocks the ambient medium.

It is important to appreciate that the $\mathrm{SN}$ light is primarily powered by radioactive decay of the freshly synthesized ${ }^{56} \mathrm{Ni}$, whereas the GRBs are powered by the activity of the central engine. In the current generation of collapsar models, there is sufficient flexibility to allow for a large dispersion of ${ }^{56} \mathrm{Ni}$ and the energy of the engine. Thus, the next phase of understanding the GRB-SN connection ${ }^{18}$ will benefit from (and require) observational measures of these parameters.

Motivated thus, we have an ongoing program of searches for SNe in GRB afterglows with the Hubble Space Telescope $(H S T)$. Here we present a systematic search for an SN underlying GRB 010921. We present our observations in $\S 2$ and the details of photometry in $\S 3$. We fit afterglow models and constrain the brightness of an underlying SN in $\S 4$. We then present an overview of previous such efforts and conclude in $\S 5$.

\section{OBSERVATIONS AND REDUCTIONS}

GRB 010921 was detected by the High Energy Transient Explorer (HETE-2) satellite on 2001 September 21.219 UT (Ricker et al. 2002), and the position was refined by the Interplanetary Network error box (Hurley et al. 2001). Using the $5 \mathrm{~m}$ Hale Telescope and the Very Large Array, we discovered the afterglow of this event as well as the redshift of the host galaxy (Price et al. 2002a).

The low redshift of this event, $z=0.451$, made it a prime candidate for a search for an underlying SN. Accordingly, as a part of our large HST Cycle 9 program (GO-8867, PI: S. R. Kulkarni), we triggered a series of observations with the Wide Field Planetary Camera 2 (WFPC2) aboard HST. Because of the lateness in identifying the afterglow candidate, the first observation was on day 35 , slightly after the expected peak of the SN. At each of epochs 1-3 we obtained $4 \times 400 \mathrm{~s}$ exposures in each of five filters (F450W, F555W, F702W, F814W, and F850LP) with a single diagonal dither by 2.5 pixels to recover the undersampled point-spread function (PSF). The fourth epoch was optimized for photometry of the host galaxy, and, accordingly, we increased the exposure time to $4 \times 1100 \mathrm{~s}$.

We used "on-the-fly" preprocessing to produce debiased, flattened images. The images were then drizzled (Fruchter \& Hook 2002) onto an image with pixels smaller than the original by a factor of 0.7 using a pixfrac of 0.8 , yielding a final pixel scale of approximately 0 ".07 pixel $^{-1}$. After rotation to a common orientation, the images were registered to the first epoch images using the centroids of

\footnotetext{
18 A class of models, known as "supranova" models, posits an SN greatly in advance (many months) of the GRB event (Vietri \& Stella 1999). The long delay was physically motivated to explain the X-ray lines as arising from a large spatial region. The observations of SNe underlying GRBs and peaking at $\sim 20$ days (e.g., GRB 011121) do not allow for such long delays.
}

common objects in the field. The typical rms registration errors were less than 0.15 drizzled pixels.

\section{HOST SUBTRACTION AND PHOTOMETRY}

The host galaxy of GRB 010921 has an integrated magnitude of $R \sim 22 \mathrm{mag}$ (Price et al. 2002a), or about $5 \mu \mathrm{Jy}$. Consequently, great care has to be taken to properly photometer the fading afterglow. Below we review various photometric techniques.

Total magnitudes.- The simplest technique is to perform aperture photometry (e.g., Galama et al. 2000; Price et al. 2001). The afterglow flux is obtained by subtracting the host flux estimated from a very late time measurement. A major concern is that the host flux is dependent on the choice of aperture (both center and size). Thus, if different images have different seeing, then it is possible to obtain an artificial bump in the light curve.

Host subtraction.-The above concern can be alleviated by subtracting a late-time image from the earlier images. The afterglow may then be easily photometered in the hostsubtracted images. This method has been used with considerable success by those observing SNe Ia (e.g., Schmidt et al. 1998).

$N(N-1) / 2$ subtraction.-In this technique, each image is subtracted from every other image and the afterglow residual photometered. The flux at each epoch can be fitted through least squares, assuming that the flux at the final epoch is zero (M. Novicki \& J. Tonry 2001, private communication $^{19}$ ). This method makes use of the fact that the host galaxy has not been observed only once at late times, but at each epoch, and thus a better signal-to-noise ratio can be obtained from the overconstrained system.

We employed the $N(N-1) / 2$ subtraction technique to photometer the GRB 010921 afterglow in our HST images. The images were subtracted using a modified version of ISIS (Alard 2000) and photometered using the analytic PSFfitting routine within Vista (J. Tonry 2001, private communication). We used the SYNPHOT package within IRAF to calculate the response of the instrument and filter combination to a source with constant flux of $1 \mathrm{mJy}$; the resulting values are AB magnitudes (Fukugita et al. 1996), expressed as fluxes. Corrections were made for charge transfer (in)efficiency (CTE) using the prescription of Dolphin (2000) and aperture corrected to infinity.

We have also reanalyzed and photometered groundbased images (Price et al. 2002a; Park et al. 2002) of the afterglow, applying $N(N-1) / 2$ subtraction. Since this technique assumes that the flux of the afterglow in the final epoch is zero, which may not be correct for these images, we subtracted the appropriate fourth-epoch HST observation (which we have assumed contains no afterglow) from the final ground-based images, measured the flux of the afterglow, and added this value to the fluxes derived from the $N(N-1) / 2$ subtraction.

The results of the photometry are host-subtracted fluxes for the afterglow in each of the images, under the assumption that the afterglow flux in the final HST image (2001 December 21) is zero (or negligible). These values are presented in Tables 1 and 2 . The values in Table 2 supersede the corresponding measurements presented in Price et al.

\footnotetext{
${ }^{19}$ See also http://www.ifa.hawaii.edu/users/mnovicki/Paper.ps.
} 
TABLE 1

HST+WFPC2 OBSERVATIONS OF GRB 010921

\begin{tabular}{ccr}
\hline $\begin{array}{c}\text { Date } \\
(2001 \mathrm{UT})\end{array}$ & Filter & \multicolumn{1}{c}{$\begin{array}{c}\text { Flux } \\
(\mu \mathrm{Jy})\end{array}$} \\
\hline Oct $26.731 \ldots \ldots \ldots \ldots$. & F450W & $-0.031 \pm 0.022$ \\
Nov $06.956 \ldots \ldots \ldots \ldots$. & F450W & $0.001 \pm 0.028$ \\
Nov $24.990 \ldots \ldots \ldots \ldots$. & F450W & $0.067 \pm 0.029$ \\
Oct $26.791 \ldots \ldots \ldots \ldots$. & F555W & $0.157 \pm 0.015$ \\
Nov $07.015 \ldots \ldots \ldots \ldots$. & F555W & $0.087 \pm 0.017$ \\
Nov $25.121 \ldots \ldots \ldots \ldots$. & F555W & $0.063 \pm 0.018$ \\
Oct $26.859 \ldots \ldots \ldots \ldots .$. & F702W & $0.231 \pm 0.013$ \\
Nov $07.149 \ldots \ldots \ldots \ldots$. & F702W & $0.096 \pm 0.015$ \\
Nov $25.203 \ldots \ldots \ldots \ldots .$. & F702W & $0.045 \pm 0.015$ \\
Oct $26.932 \ldots \ldots \ldots \ldots .$. & F814W & $0.433 \pm 0.024$ \\
Nov $08.359 \ldots \ldots \ldots \ldots .$. & F814W & $0.209 \pm 0.024$ \\
Nov $25.621 \ldots \ldots \ldots \ldots .$. & F814W & $-0.003 \pm 0.025$ \\
Oct $26.992 \ldots \ldots \ldots \ldots .$. & F850LP & $0.471 \pm 0.092$ \\
Nov $08.418 \ldots \ldots \ldots \ldots$. & F850LP & $0.207 \pm 0.088$ \\
Nov $25.687 \ldots \ldots \ldots \ldots .$. & F850LP & $0.030 \pm 0.096$ \\
\hline
\end{tabular}

NoTE.-These host-subtracted measurements have not been corrected for Galactic extinction and are all made under the assumption that the flux of the OT on 2001 December 21 is zero or negligible, $\ll 0.01 \mu \mathrm{Jy}$.

TABLE 2

REANALysis of GRound-BASEd ObSERvations of GRB 010921

\begin{tabular}{|c|c|c|c|}
\hline $\begin{array}{c}\text { Date } \\
(2001 \mathrm{UT})\end{array}$ & Filter & $\begin{array}{l}\text { Flux } \\
(\mu \mathrm{Jy})\end{array}$ & Telescope \\
\hline Oct $19.178 .$. & $g^{\prime}$ & $0.671 \pm 0.097$ & P200 \\
\hline Sep $22.144 \ldots \ldots \ldots \ldots \ldots$ & $r^{\prime}$ & $46.104 \pm 0.722$ & P200 \\
\hline Sep $22.148 \ldots \ldots \ldots \ldots \ldots$ & $r^{\prime}$ & $44.995 \pm 0.661$ & P200 \\
\hline Sep $27.354 \ldots \ldots \ldots \ldots \ldots$ & $r^{\prime}$ & $2.13 \pm 1.223$ & P200 \\
\hline Oct $17.145 \ldots \ldots \ldots \ldots \ldots$ & $r^{\prime}$ & $0.086 \pm 0.379$ & P200 \\
\hline Oct $18.088 \ldots$ & $r^{\prime}$ & $0.189 \pm 0.382$ & P200 \\
\hline Oct $19.109 .$. & $r^{\prime}$ & $0.256 \pm 0.285$ & P200 \\
\hline Oct $17.165 \ldots$ & $i^{\prime}$ & $0.560 \pm 0.197$ & P200 \\
\hline Oct $18.110 \ldots .$. & $i^{\prime}$ & $0.523 \pm 0.191$ & P200 \\
\hline Oct $19.130 \ldots \ldots$ & $i^{\prime}$ & $0.649 \pm 0.153$ & P200 \\
\hline Oct $19.149 \ldots$. & $z^{\prime}$ & $1.293 \pm 4.273$ & P200 \\
\hline Sep $22.3038 .$. & $B$ & $11.319 \pm 0.981$ & NOFS1.0 \\
\hline Oct $19.253 \ldots$ & $B$ & $0.623 \pm 0.675$ & P60 \\
\hline Sep 22.2976 . & $V$ & $24.727 \pm 1.078$ & NOFS1.0 \\
\hline Oct $19.206 \ldots .$. & $V$ & $0.229 \pm 0.720$ & P60 \\
\hline Sep 22.2930 . & $R$ & $39.116 \pm 5.072$ & NOFS1.0 \\
\hline Sep $22.3210 \ldots \ldots \ldots \ldots . .$. & $R$ & $36.135 \pm 4.486$ & NOFS1.0 \\
\hline Oct $19.272 \ldots \ldots \ldots \ldots \ldots$ & $R$ & $0.916 \pm 4.284$ & P60 \\
\hline Nov $17.151 \ldots \ldots \ldots \ldots . . . . .$. & $R$ & $0.470 \pm 4.238$ & NOFS1.0 \\
\hline Sep $22.2893 \ldots \ldots \ldots \ldots . .$. & $I$ & $84.688 \pm 5.778$ & NOFS1.0 \\
\hline Sep $22.795 \ldots \ldots \ldots \ldots \ldots$ & $I$ & $40.277 \pm 3.950$ & TS \\
\hline Sep $22.825 \ldots \ldots \ldots \ldots \ldots$ & $I$ & $47.281 \pm 7.230$ & TS \\
\hline Sep $22.878 \ldots \ldots \ldots \ldots \ldots$ & $I$ & $50.926 \pm 3.671$ & TS \\
\hline Sep $22.954 \ldots \ldots \ldots \ldots \ldots$ & $I$ & $41.321 \pm 3.636$ & TS \\
\hline Nov $17.093 \ldots \ldots \ldots \ldots . . .$. & $I$ & $1.229 \pm 1.057$ & NOFS1.0 \\
\hline
\end{tabular}

Note.-These host-subtracted measurements have not been corrected for Galactic or host extinction and are all made under the assumption that the flux of the OT on 2001 December 21 is zero or negligible. Zero points were set from the star at coordinates R.A. $=22^{\mathrm{h}} 56^{\mathrm{m}} 00^{\mathrm{s}} \cdot 21$, decl. $=40^{\circ} 54^{\prime} 58^{\prime \prime} .0$ with $B=21.248$ mag, $V=20.230 \mathrm{mag}, R=19.699 \mathrm{mag}$, and $I=19.132 \mathrm{mag}$, accurate to better than 3\% (Henden 2001). Telescopes are as follows: P200Hale Palomar 200 inch; NOFS1.0 - USNO Flagstaff Station 1.0 m; P60-Palomar 60 inch; TS - Tautenburg Schmidt. NOFS1.0 observations of September 22 and all P200 and P60 observations were presented in Price et al. 2002a; NOFS1.0 and TS observations were presented in Park et al. 2002.

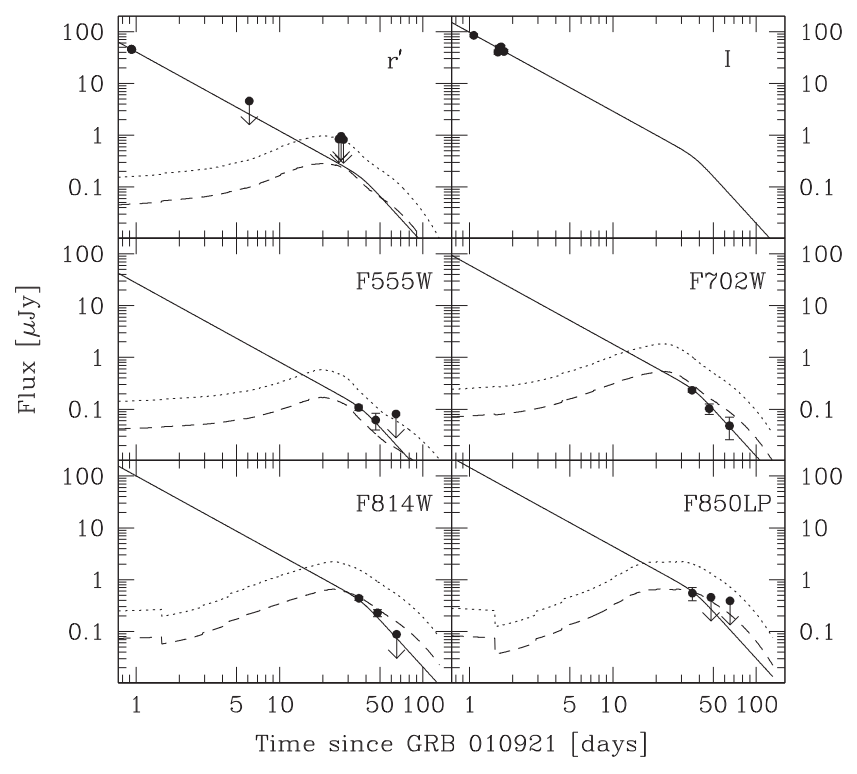

FIG. 1.-Optical light curves of the afterglow of GRB 010921. Each data point contains pure afterglow (no contribution from the host galaxy) and has not been corrected for foreground extinction. Downward-pointing arrows indicate $2 \sigma$ upper limits. The fit is a standard broken power-law afterglow model $(\S 4)$. The dotted line is the light curve of SN 1998bw shifted to $z=0.451$ and placed behind the same column as the GRB; the dashed line is the same as the dotted line, but dimmed by 1.33 mag (our $3 \sigma$ sensitivity).

(2002a) and Park et al. (2002). We plot the afterglow light curves in Figure 1. The light curves are monotonically decreasing (i.e., do not level off), and fits to the afterglow measurements $(\S 4)$ all yield fluxes at the epoch of our final HST images that are below the detection limit; hence, we deduce that our assumption of negligible flux in the final HST image is justified.

\section{DISCUSSION}

Temporal breaks in optical light curves have been seen in many afterglows and are usually attributed to a "jet" geometry (see Frail et al. 2001). We adopt a standard optical afterglow model, consisting of a broken power-law temporal decay with power-law indices $\alpha_{1}$ and $\alpha_{2}$ and a power-law spectral index $\beta$ (Sari, Piran, \& Narayan 1998; Sari, Piran, \& Halpern 1999).

Each of these indices is a function of the electron energy distribution index, $p$, dependent on the location of the cooling break relative to the optical bands, and so we consider two cases: the cooling break is redward of the optical (hereafter case R), and the cooling break is blueward of the optical (case B). We consider, in addition to a constant circumburst medium, an inhomogeneous circumburst medium, $\rho \propto r^{-2}$ (see Chevalier \& Li 2000; Price et al. 2002b).

We apply the parametric extinction curves of Cardelli, Clayton, \& Mathis (1989) and Fitzpatrick \& Massa (1988) using the interpolation calculated by Reichart (2001). These extinction curves are characterized by two values: the magnitude of the extinction in the rest frame of the host galaxy, $A_{V}^{\text {host }}$, and the slope of the UV extinction curve, $c_{2}$ (see Reichart 2001). Following Price et al. (2002a), we adopt $c_{2}=4 / 3$, corresponding to an LMC-like extinction curve. 
Adopting other extinction curves (e.g., Milky Way, SMC) yields similar but more-constraining results (i.e., any underlying SN must be even fainter than the upper limit we derive below; see Price et al. 2002a).

Observationally, an excess of red emission ("red bumps") at late times has been established in some GRBs and has been reasonably interpreted as arising from an underlying SN. However, to demonstrate that these bumps are definitively of SN origin requires spectroscopic observations in which SN features are seen. Given the current quality of the data (even for GRB 011121), it is premature to start "typing" these bumps. On theoretical grounds, one expects $\mathrm{SNe} \mathrm{Ib} / \mathrm{c}$, but theoretical models (see MacFadyen, Woosley, \& Heger 2001) simply lack the detail to predict the expected light curves.

Given this discussion and the absence of a red bump in the afterglow of GRB 010921, we now carry out a restricted analysis for an underlying SN in the context of the collapsar model. To this end, we use the observations of SN 1998bw for an SN template since it is one of the well-observed bright $\mathrm{SNe} \mathrm{Ib} / \mathrm{c}$ that may be related to a GRB (Galama et al. 1998). ${ }^{20}$ Specifically, we used the UBVRI photometry of Galama et al. (1998) and derived the flux distribution of SN $1998 \mathrm{bw}$, using the zero points and filter curves of Bessell (1990). The resulting low-resolution spectrum (consisting of five points at the effective wavelength of each broadband filter) is redshifted to $z=0.451$ (Price et al. 2002a), assuming a flat lambda cosmology with $\Omega_{M}=0.3$ and $H_{0}=65 \mathrm{~km}$ $\mathrm{s}^{-1} \mathrm{Mpc}^{-1}$. The redshifted spectrum, which represents what SN 1998bw would look like at cosmological distances, is integrated with the appropriate filter curve to derive the apparent brightness at this redshift.

SN 1998 bw at $z=0.451$ would peak in the rest-frame $I$ band at approximately $4 \mu \mathrm{Jy}$. It is evident from Figure 1 that the afterglow is much fainter than this and, further, that there is no clear bump in the afterglow light curve. We therefore allow the $\mathrm{SN}$ component to be scaled by $\delta$ magnitudes in our model. The $\mathrm{SN}$ is placed behind the same foreground (i.e., Milky Way) and host galaxy extinction as the afterglow (which can be inferred by demanding that the temporal and intrinsic spectral slopes, which both depend on the electron distribution index, $p$, be consistent; see, e.g., Price et al. 2002b).

To calculate the SN detection limit of our observations, we fitted the model by minimizing $\chi^{2}$. The afterglow was not detected in any of the F450W images, and so we exclude them from our analysis. Subtracting the host F450W image from our ground-based $g^{\prime}$ image left a large $(\sim 10 \sigma)$ residual at the position of the host galaxy (not of the optical transient [OT]). This poor subtraction is likely due to the filter mismatch and the bands being affected by the Balmer break, so we do not include this point in our analysis.

Our analyses are summarized in Table 3 . In short, we find no evidence for an underlying SN. In order to calculate the formal limits, we refitted the data for a range of values of the SN brightness and computed the probability distribution from the resultant $\Delta \chi^{2}$. As can be seen from Table 4 , the least constraining limit comes from the case in which the afterglow evolves in a wind-stratified medium with the

${ }^{20}$ We note that our observations will be even more sensitive to $\mathrm{SNe}$ with long-lived tails (e.g., Type II-P) and would therefore yield stringent limits for these types of SNe as well.
TABLE 3

Best-Fit Afterglow Models

\begin{tabular}{ccccc}
\hline \hline Model & $p$ & $\begin{array}{c}t_{\text {jet }} \\
\text { (days) }\end{array}$ & $\begin{array}{c}A_{V}^{\text {host }} \\
(\mathrm{mag})\end{array}$ & $\chi^{2}$ \\
\hline ISM/wind, B....... & $2.67 \pm 0.06$ & $33.0 \pm 6.5$ & $0.95 \pm 0.08$ & 19.9 \\
ISM, R ............... & $3.03 \pm 0.04$ & $37.5 \pm 4.9$ & $1.16 \pm 0.07$ & 19.2 \\
Wind, R ............. & $2.33 \pm 0.10$ & $30.3 \pm 9.5$ & $1.35 \pm 0.08$ & 23.1 \\
\hline
\end{tabular}

NoTE.-The best-fit afterglow parameters from fitting a standard afterglow model with host extinction and no SN. Each fit had 32 degrees of freedom. ISM models refer to afterglow evolution in a homogeneous interstellar medium. Wind models refer to afterglow evolution in a windstratified $\left(r^{-2}\right)$ medium. $\mathrm{R}$ and $\mathrm{B}$ refer to the location of the cooling break relative to the optical bands (redward and blueward, respectively). If the cooling break is blueward of the optical bands, then the ISM and wind models both have the same form.

cooling break redward of the optical band, and even in this case, an SN brighter than $\delta=1.33$ mag is excluded at $99.7 \%$ confidence, and an SN as bright as SN 1998bw ( $\delta=0$ mag) is ruled out at greater than $99.999 \%$ confidence. We note that this result is largely independent of the exact shape of the assumed SN light curve, since our primary constraint is on the flux level, but an earlier peak time for the SN may render our observations (taken relatively late) less constraining.

The peak brightness and the timescales for $\mathrm{SNe} \mathrm{Ib/c} \mathrm{are}$ generally correlated such that fainter SNe may peak earlier (Iwamoto et al. 1998). It may be important to take this into account for our analysis, since the observations most sensitive to the presence of an underlying SN are all after the peak. To do this, we shifted the UBVRI photometry of the (intrinsically) fainter Type Ic SN 1994I (Richmond et al. 1996) to $z=0.451$ and derived the transformation between the redshifted SN 1998bw and SN 1994I light curves using a similar method as Bloom et al. (2002b). This method is analogous to the "stretch" method for SNe Ia (Perlmutter et al. 1999). If we use this transformation in our model to transform the redshifted SN 1998bw light curve to the light curve of an SN fainter than SN 1998 bw by $\delta$ magnitudes, then our least constraining limit on an underlying $\mathrm{SN}$ is still $\delta=1.33$ mag fainter than SN 1998bw (at 99.7\% confidence). The agreement with the above limit indicates that the uncertainty in our knowledge of the light-curve shape and luminosity scaling light curve is not important for this analysis.

Leaving aside the $\mathrm{SN}$ issue, our fits provide a jet break time of approximately 35 days. From the FREGATE $8-400$ $\mathrm{keV}$ fluence of $1.5 \times 10^{-5} \mathrm{ergs} \mathrm{cm}^{-2}$, we calculate the $k$-corrected isotropic-equivalent energy release (Bloom, Frail, \& Sari 2001) in the gamma-ray band, $E_{\gamma} \sim(1.3 \pm 0.3) \times 10^{52}$

TABLE 4

Maximum Allowed Brightness of an SN Underlying GRB 010921

\begin{tabular}{cccc}
\hline \hline Significance & ISM/Wind, B & ISM, R & Wind, R \\
\hline $1 \sigma(68.3 \%) \ldots \ldots \ldots$ & $2.887(2.887)$ & $2.651(2.614)$ & $2.387(2.387)$ \\
$2 \sigma(95.4 \%) \ldots \ldots \ldots$ & $2.166(2.166)$ & $1.950(1.937)$ & $1.726(1.705)$ \\
$3 \sigma(99.7 \%) \ldots \ldots \ldots$ & $1.721(1.721)$ & $1.549(1.549)$ & $1.333(1.333)$ \\
\hline
\end{tabular}

Note.-For each model and significance level, we list the magnitude relative to SN 1998bw of the faintest SN detectable by the observations. We include in parentheses the magnitude relative to SN 1998bw of a "generic SN." Model descriptions are the same as in Table 3. 
ergs. Applying the geometric correction from our measurement of the jet break (using the formulation and normalization of Frail et al. 2001), we obtain a jet opening angle of $18^{\circ}$. Thus, the true energy release is $(6.5 \pm 1.6) \times 10^{50} \mathrm{ergs}$, consistent with the clustering of energy releases around $5 \times 10^{50}$ ergs (Frail et al. 2001)

\section{CONCLUSIONS}

Here we report the search for an underlying $\mathrm{SN}$ in the afterglow of GRB 010921. Thanks to the superb photometric stability of $H S T$ and the $N(N-1) / 2$ subtraction technique, we have been able to trace the light curve of the afterglow of GRB 010921 over 2 months. The resulting photometry is unbiased by aperture effects that are so prevalent in simple aperture and PSF-fitting photometry. We report two results.

First, we find a jet break time of 35 days, using only optical data. Second, we find no evidence for an SN. An SN, if present, must be fainter than SN 1998bw by greater than $1.33 \mathrm{mag}$ at $99.7 \%$ confidence. To our knowledge, to date, this is the most stringent limit for an underlying SN associated with a cosmologically located GRB.

As noted in $\S 1$, the collapsar model as currently understood has little power in predicting the dispersion in the amount of ${ }^{56} \mathrm{Ni}$ synthesized as compared to the energy in relativistic ejecta. Underlying $\mathrm{SNe}$ are directly powered by the former, whereas the GRB is powered by the latter. Observations are needed to start mapping the distribution in these critical explosion parameters. Progress can be expected with such observational inputs accompanied by further refinements in the model. Motivated thus, we summarize in Table 5 the status of SN searches for all known GRBs with redshift ${ }^{21}$ less than 1.2.

The most secure case for an $\mathrm{SN}$ is that for GRB 011121 (Bloom et al. 2002b; Garnavich et al. 2002). GRB 980326 shows a strong red excess at about a month, but unfortu-

${ }^{21}$ Beyond a redshift of $\sim 1.2$, the distinctive and strong absorption blueward of $4000 \AA$ is redshifted out of the optical bands. The higher sensitivity of the optical bands thus favors searches for SNe below this redshift. nately a redshift is lacking. GRB 970228 shows a less clear excess but benefits from a known redshift. Stated conservatively, an SN as bright as that of SN 1998bw can be ruled out in GRB 000911. In all cases, save that of GRB 980326 and GRB 011121, the presence of a host with a magnitude comparable to the brightness of the peak of the SN makes it difficult to identify an SN component. As noted in $\S 3$, "bumps" can arise from host contamination. Combining HST and ground-based measurements (as is the case for GRB 970228) is prone to considerable errors ( $(3)$.

In summary, there is good evidence for an SN comparable in brightness to SN 1998bw in GRB 011121 (Bloom et al. 2002b; Garnavich et al. 2002). For GRB 010921, using the HST observations reported here, we constrain any putative underlying $\mathrm{SN}$ to be $1.33 \mathrm{mag}$ fainter than SN 1998bw. In the collapsar framework, this absence could be most readily attributed to the well-known dispersion of the peak luminosity of SNe Ib/c (Clocchiatti et al. 2000).

An alternative possibility is that there may be more than one type of progenitor for long-duration GRBs. Along these lines we note that Chevalier \& Li (2000) claim that some afterglows (e.g., GRB 990123) are incompatible with a $\rho \propto r^{-2}$ inhomogeneous circumburst distribution whereas other afterglows (e.g., GRB 970228 and GRB 970508) are better explained by invoking an inhomogeneous circumburst medium. Progress requires both searches for underlying $\mathrm{SNe}$ as well as characterizing the circumburst medium via modeling of the early-time afterglow (e.g., GRB 011121; see Price et al. 2002b).

Finally, we note that the afterglow of GRB 010921 (and any coincident $\mathrm{SN}$ ) was extincted by $A_{V}^{\mathrm{MW}} \approx 0.5 \mathrm{mag}$ of dust in the foreground and $A_{V}^{\text {host }} \approx 1$ mag of dust in the host galaxy (Table 3). Thus, in the future, using ACS aboard $H S T$, it should be possible to extend SN searches to at least 3 mag fainter than SN 1998bw, at which point it will be possible to detect more typical SNe Ib/c coincident with GRBs.

We thank Pete Challis for helpful discussions about WFPC2 reduction and Megan Novicki and John Tonry for an advance copy of their $N(N-1) / 2$ subtraction paper. S. R. K. and S. G. D. thank NSF for supporting our

TABLE 5

Status of SN Searches for All Known GRBs with $z<1.2$

\begin{tabular}{|c|c|c|c|c|c|c|c|}
\hline GRB & $z$ & Band & $\begin{array}{c}\mathrm{SN} \\
(\mathrm{mag})\end{array}$ & $\begin{array}{l}\text { Host } \\
\text { (mag) }\end{array}$ & Ground/HST? & Comments & References \\
\hline $970228 \ldots \ldots \ldots . . .$. & 0.695 & $R$ & 25.5 & 25.2 & Both & Plausible but aperture, color effects with $H S T$ & 1 \\
\hline $970508 \ldots \ldots \ldots . .$. & 0.835 & $I$ & 23.6 & 24.0 & Ground & Aperture effects & 2 \\
\hline $980326 \ldots \ldots \ldots$. & $? ? ?$ & $R$ & 25 & $>27$ & Ground & Plausible & 3 \\
\hline $980613 \ldots \ldots \ldots$ & 1.096 & $R$ & $\ldots$ & 24.0 & Ground & Faint afterglow, no search & 4 \\
\hline $980703 \ldots \ldots \ldots$. & 0.966 & $R$ & 24 & 22.6 & Ground & $\mathrm{SN} /$ no SN equally consistent & 5 \\
\hline $990705 \ldots \ldots \ldots . . .$. & 0.840 & $R$ & $\ldots$ & 22.8 & Ground & No search & \\
\hline $990712 \ldots \ldots \ldots . .$. & 0.433 & $V$ & 23.8 & 21.2 & Ground & Aperture effects? & 6 \\
\hline $991208 \ldots \ldots \ldots . . .$. & 0.706 & $R$ & 23.9 & 24.4 & Ground & $\chi^{2} / \mathrm{dof}=1.8$ & 7 \\
\hline $991216 \ldots \ldots \ldots . .$. & 1.020 & $R$ & $\ldots$ & 24.85 & Ground & No search, consistent with no SN & 8 \\
\hline $000418 \ldots \ldots \ldots$ & 1.119 & $R$ & $\ldots$ & 23.8 & Ground & Consistent with no SN & 9 \\
\hline $000911 \ldots \ldots \ldots$ & 1.058 & $I$ & 24.7 & 24.4 & Ground & $2 \sigma$ detection, $\mathrm{SN} \sim(0.9 \pm 0.3) \mathrm{SN} 1999 \mathrm{bw}$ & 10 \\
\hline $011121 \ldots \ldots \ldots$ & 0.365 & $R$ & 23 & 26 & $H S T$ & Secure: "SN 2001ke" & 11 \\
\hline
\end{tabular}

Note.-All GRBs with optical afterglows at $z<1.2$, excepting GRB 980425 (SN 1998bw) and GRB 010921 (this study). Host magnitudes and redshifts were also compiled from Djorgovski et al. $2001 \mathrm{~b}$.

REFERENCES.- (1) Reichart 1999; Galama et al. 2000. (2) Sokolov 2001. (3) Bloom et al. 1999. (4) Hjorth et al. 1999. (5) Holland et al. 2001. (6) Björnsson et al. 2001. (7) Castro-Tirado et al. 2001. (8) Garnavich et al. 2000; Halpern et al. 2000. (9) Berger et al. 2001. (10) Lazzati et al. 2001. (11) Bloom et al. 2002b. 
ground-based GRB observing program. B. P. S. and P. A. P. thank the ARC for supporting Australian GRB research. Support for proposal HST-GO-08867.01-A was provided by NASA through a grant from the Space Telescope Science
Institute, which is operated by the Association of Universities for Research in Astronomy, Inc., under NASA contract NAS 5-26555. K. H. is grateful for support under grant HST-GO-09180.07-A.
Alard, C. 2000, A\&AS, 144, 363

Berger, E., Kulkarni, S. R., \& Frail, D. A. 2001a, ApJ, 560, 652

Berger, E., et al. 2001b, ApJ, 556, 556

Bessell, M. S. 1990, PASP, 102, 1181

Björnsson, G., Hjorth, J., Jakobsson, P., Christensen, L., \& Holland, S. 2001, ApJ, 552, L121

Bloom, J. S., Frail, D. A., \& Sari, R. 2001, AJ, 121, 2879

Bloom, J. S., Kulkarni, S. R., \& Djorgovski, S. G. 2002a, AJ, 123, 111

Bloom, J. S., et al. 1999 , Nature, 401, 453 2002 b, ApJ, 572, L45

Cardelli, J. A., Clayton, G. C., \& Mathis, J. S. 1989, ApJ, 345, 245

Castro-Tirado, A. J., et al. 2001, A\&A, 370, 398

Chevalier, R. A., \& Li, Z. 2000, ApJ, 536, 195

Clocchiatti, A., et al. 2000, ApJ, 529, 661

Djorgovski, S. G., Frail, D. A., Kulkarni, S. R., Bloom, J. S., Odewahn, S. C., \& Diercks, A. 2001a, ApJ, 562, 654

Djorgovski, S. G., et al. 2001b, in Gamma-Ray Bursts in the Afterglow Era, ed. E. Costa, F. Frontera, \& J. Hjorth (Heidelberg: Springer), 218

Dolphin, A. E. 2000, PASP, 112, 1397

Fitzpatrick, E. L., \& Massa, D. 1988, ApJ, 328, 734

Frail, D. A., et al. 2001, ApJ, 562, L55 2002, ApJ, 565, 829

Fruchter, A. S., \& Hook, R. N. 2002, PASP, 114, 144

Fukugita, M., Ichikawa, T., Gunn, J. E., Doi, M., Shimasaku, K., \& Schneider, D. P. 1996, AJ, 111, 1748

Galama, T. J., \& Wijers, R. A. M. J. 2001, ApJ, 549, L209

Galama, T. J., et al. 1998, Nature, 395, 670 2000, ApJ, 536, 185

Garnavich, P. M., Jha, S., Pahre, M. A., Stanek, K. Z., Kirshner, R. P.,

Garcia, M. R., Szentgyorgyi, A. H., \& Tonry, J. L. 2000, ApJ, 543, 61

Garnavich, P. M., et al. 2002, ApJ, submitted

Halpern, J. P., et al. 2000, ApJ, 543, 697

Harrison, F. A., et al. 2001, ApJ, 559, 123

\section{REFERENCES}

Henden, A. 2001, GCN Circ. 1100 (http://gcn.gsfc.nasa.gov/gcn/gcn3/ $1100 . \operatorname{gcn} 3)$

Hjorth, J., Pedersen, H., Jaunsen, A. O., \& Andersen, M. I. 1999, A\&AS 138,461

Holland, S., et al. 2001, A\&A, 371, 52

Hurley, K., et al. 2001, GCN Circ. 1097 (http://gcn.gsfc.nasa.gov/gcn/ $\operatorname{gcn} 3 / 1097 . \mathrm{gcn} 3$ )

Iwamoto, K., et al. 1998, Nature, 395, 672

Kulkarni, S. R. et al. 1998, Nature, 395, 663

Lazzati, D., et al. 2001, A\&A, 378, 996

MacFadyen, A. I., \& Woosley, S. E. 1999, ApJ, 524, 262

MacFadyen, A. I., Woosley, S. E., \& Heger, A. 2001, ApJ, 550, 410

Park, H. S., et al. 2002, ApJ, 571, L131

Perlmutter, S, et al. 1999, ApJ, 517, 565

Piro, L., et al. 2000, Science, 290, 955 2002, ApJ, 577, 680

Price, P. A., et al. 2001, ApJ, 549, L7 2002a, ApJ, 571, L12 2002 b, ApJ, 572, L 51

Reeves, J. N., et al. 2002, Nature, 416, 512

Reichart, D. E. 1999, ApJ, 521, L111 2001, ApJ, 553, 235

Richmond, M. W., et al. 1996, AJ, 111, 327

Ricker, G., et al. 2002, ApJ, 571, L127

Sari, R., Piran, T., \& Halpern, J. P. 1999, ApJ, 519, L17

Sari, R., Piran, T., \& Narayan, R. 1998, ApJ, 497, L17

Schmidt, B. P., et al. 1998, ApJ, 507, 46

Sokolov, V. V. 2001, in Gamma-Ray Bursts in the Afterglow Era, ed. E. Costa, F. Frontera, \& J. Hjorth (Heidelberg: Springer), 136

Stanek, K. Z., Garnavich, P. M., Holland, S. T., Jha, S., Kirshner, R. P., \& Bersier, D. 2002, IAU Circ., 7857, 2

Vietri, M., \& Stella, L. 1999, ApJ, 527, L43

Woosley, S. E. 1993, ApJ, 405, 273 